\title{
Journal of 3D Printing in Medicine
}

\section{Antibiotics in 3D-printed implants, instruments and materials: benefits, challenges and future directions}

\author{
David H Ballard*,1(D), Karthik Tappa' (iD), Christen J Boyer², Udayabhanu Jammalamadaka', \\ Kavya Hemmanur ${ }^{1}$, Jeffery A Weisman ${ }^{3}$, Jonathan S Alexander ${ }^{2}$, David K Mills ${ }^{4}$ \& Pamela K \\ Woodard ${ }^{1}$ \\ ${ }^{1}$ Mallinckrodt Institute of Radiology, Washington University School of Medicine, St Louis, MO 63110, USA \\ ${ }^{2}$ Department of Molecular \& Cellular Physiology, Louisiana State University Health Shreveport, LA 71103, USA \\ ${ }^{3}$ University of Illinois at Chicago Occupational Medicine, Chicago, IL 60612, USA \\ ${ }^{4}$ Department of Biomedical Engineering, Louisiana Tech University, Ruston, LA 71272, USA \\ *Author for correspondence: Tel.: +1 314226 5464; Fax: +1 314747 4671; davidballard@wustl.edu
}

3D printing is an additive manufacturing technology, which permits innovative approaches for incorporating antibiotics into 3D printed constructs. Antibiotic-incorporating applications in medicine have included medical implants, prostheses, along with procedural and surgical instruments. 3D-printed antibioticimpregnated devices offer the advantages of increased surface area for drug distribution, sequential layers of antibiotics produced through layer-by-layer fabrication, and the ability to rapidly fabricate constructs based on patient-specific anatomies. To date, fused deposition modeling has been the main 3D printing method used to incorporate antibiotics, although inkjet and stereolithography techniques have also been described. This review offers a state-of-the-art summary of studies that incorporate antibiotics into 3Dprinted constructs and summarizes the rationale, challenges, and future directions for the potential use of this technology in patient care.

First draft submitted: 26 March 2019; Accepted for publication: 23 April 2019; Published online: 31 May 2019

Keywords: 3D printing • additive manufacturing • antibiotics $\bullet$ patient specific medicine $\bullet$ personalized medicine

3D printing technologies, namely fused deposition modeling (FDM), allow for incorporation of bioactive materials into printed constructs. This collective technique is sometimes referred to as bioactive printing or bioactive 3D printing [1]. Bioactive printing with antibiotic-impregnated constructs has been the focus of several investigations [227], the majority of which are in vitro studies [2-4,8-18]. This promising technology allows for patient-specific medicine with customization of the implant, instrument or other construct, tailoring the concentration of antibiotic, optimizing the placement and distribution of antibiotic within the construct, with rapid assembly. Although there are many potential benefits, feasibility of using antibiotic-impregnated 3D-printed constructs has primarily been demonstrated through in vitro cell culture studies, with fewer animal studies [5-7]. The few animal studies have focused only on treatment of osteomyelitis. Further validation with animal and human studies is still needed and there are a number of challenges to use these customized implants and instruments in patient care. The purpose of the present work is to offer a state-of-the-art summary of the most current investigations that incorporate antibiotics into 3D-printed constructs and summarize the rationale, challenges, and future directions for their potential use of this technology in patient care.

\section{Rationale of incorporating antibiotics into 3D-printed constructs}

Systemic antibiotics can have unintended adverse drug reactions, and higher concentrations are limited by drugs' excretion profiles and half-lives. An antibiotic-containing implant placed at the nidus of infection or site at risk for infection allows for targeted localized drug delivery that may be given at a higher concentration than a systemic dose. Because synthetic implants of any kind lack blood and lymphatic supplies, the interstitial flow and oxygen delivery are low within them; this often creates a highly hospitable environment for many pathogens. While many 


\begin{tabular}{|c|c|c|c|c|}
\hline Process & Inkjet & Fused deposition modeling & Stereolithography & Ref. \\
\hline Material choice & Limited & Wide variety & Limited & - \\
\hline $\begin{array}{l}\text { Thermal degradation of added } \\
\text { antibiotic }\end{array}$ & $\begin{array}{l}\text { Only if postprocessing involves } \\
\text { heating }\end{array}$ & Possible & Not applicable & {$[36,37]$} \\
\hline $\begin{array}{l}\text { Ultraviolet degradation of } \\
\text { added antibiotic }\end{array}$ & Not applicable & Not applicable & $\begin{array}{l}\text { Possible if drugs used are } \\
\text { ultraviolet sensitive }\end{array}$ & - \\
\hline $\begin{array}{l}\text { Mechanical reduction with } \\
\text { adding antibiotic }\end{array}$ & None in one study & Possible & Unknown & {$[2,37]$} \\
\hline
\end{tabular}

approaches have sought to encourage vascularization to permit normal immune surveillance, until this vascular supply is adequate, synthetic materials are always a 'risk' for developing into a nidus of infection. Consequently, the introduction of antibiotics into the composition of these devices may minimize risks/hazards associated with implantation of these materials and devices $[28,29]$.

The current strategy of creating antibiotic-impregnated or containing implants is most commonly through de novo intraoperative mixing or preformed antibiotic bead production [30]. Alternatively, coating implants with drugs including antibiotics used in clinical practice may be achieved through intermixing drugs into porous structures, bonding the drug directly to the surface of the implant material, or through adding a membranous drug-containing coating [31]. The use of antibiotic-impregnated implants was first reported in 1970 [32] and continues to be used in a wide variety of indications with antibiotic-impregnated beads and cement, in conditions such as osteomyelitis, soft tissue infections or as packing material for dead space. Its mechanism of action is slow elution of the embedded antibiotics as the material dissolves over time [30].

Incorporating antibiotics onto a dissolvable coat or having them bound to the superficial surface of an implant releases the antibiotic within the implanted tissue, degrading over time with the majority, if not all, of the coated or superficially bound antibiotic exposed to its environment. Alternatively, intermixing antibiotics within the internal base material of the implant or incorporating the drug into the porous structures of the material often allows for the highest concentration of drug or distributing the impregnated antibiotic over the greatest surface area [31]. 3D printing has the additional advantage of adding multiple drugs that may interfere with bone cement formation. In one animal study, antibiotic-impregnated 3D-printed implants showed a significantly reduced bacterial burden compared with antibiotic-impregnated bone cement [5].

The potential advantage of building a 3D-printed construct impregnated with antibiotics is that the drug is present at deep layers that are not initially exposed to the tissue upon initial implant (Figure 1). Additional advantages include the layer-by-layer fabrication which leads to increased surface area for drug distribution and the ability to rapidly fabricate constructs based on patient-specific anatomy derived from medical imaging. An example of incorporation of antibiotics into the structure of a 3D-printed construct is shown in Figure 2.

\section{Technical considerations}

3D printing techniques including inkjet, FDM, and stereolithography allow for highly customizable configurations of antibiotic impregnated 3D printed constructs, permitting patient-specific implants which facilitate personalized medicine. A variety of methods to incorporate antibiotics into polymers using $3 \mathrm{D}$ printing technologies have been investigated [2-27]. The earliest reports used inkjet printing [2,3], whereas the majority of subsequent studies have used FDM [7-23], and a few studies have used stereolithography [24-27].

The advantages and disadvantages of the three different 3D printing methods previously used to incorporate antibiotics into 3D-printed constructs are summarized in Table 1. In inkjet printing, the powder base material and the liquid binder must be compatible with each other to form a strong bond and hold shape, which limits the number of materials that can be used. The printing process itself does not typically use heat; however, postprocessing techniques may use heating. Therefore, inkjet printing can use a variety of antibiotics without thermal degradation (unless the postprocessing techniques use heating) but the number of base materials are limited. Stereolithography uses photocuring of liquid resins by light sources (often ultraviolet), thus is limited to materials that can be polymerized using light sources. Antibiotics that are sensitive to ultraviolet degradation may undergo degradation. Thus, stereolithography is limited in the number of base materials and drugs that are insensitive to ultraviolet radiation [33-36]. 


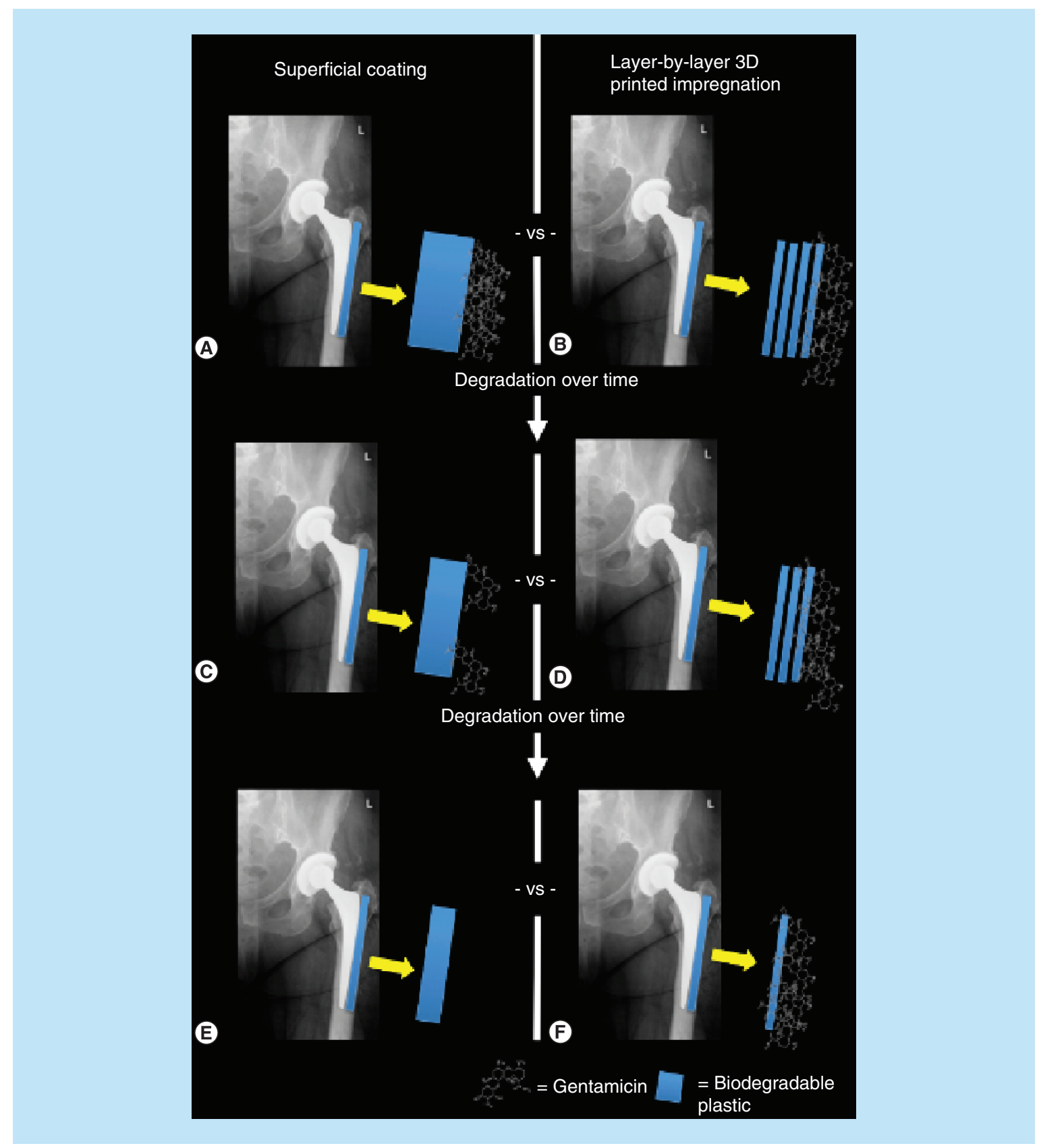

Figure 1. Drug elution characteristics of superficial coatings versus 3D-printed impregnation of antibiotics into medical implants, illustrating the advantage of 3D-printed layer-by-layer impregnation compared with a superficial coating of antibiotic. (A-F) Left hip radiograph of a hip arthroplasty with an illustrative diagram of a biodegradable plastic (illustrated in blue) superimposed on its femoral component with a superficial coating (A, C \& E) and 3D-printed impregnated technique of an antibiotic (gentamicin, illustrated by compound). Sequential images in (A \& B), (C \& D), and (E \& F) show degradation of the biodegradable plastic over time and the effect of the impregnated antibiotic. As the superficial plastic degrades, the drug concentration exposed to the environment decreases (A, C \& E). In contrast, as the 3D-printed antibiotic-impregnated plastic degrades over time, a new layer of drug is exposed to the surface thereby exposing previously unavailable antibiotic to the environment.

FDM, also called 'hot melt extrusion', is a type of 3D printing technique that heats polymers to subsequently print them and offers the advantage of a wide variety of base materials. Incorporating drugs in FDM techniques often involves processing at high temperatures and thus there is a chance of decrease in bioactivity and potential for chemical by-products through thermal degradation. Thus, thermolabile drugs cannot be used in this type of 

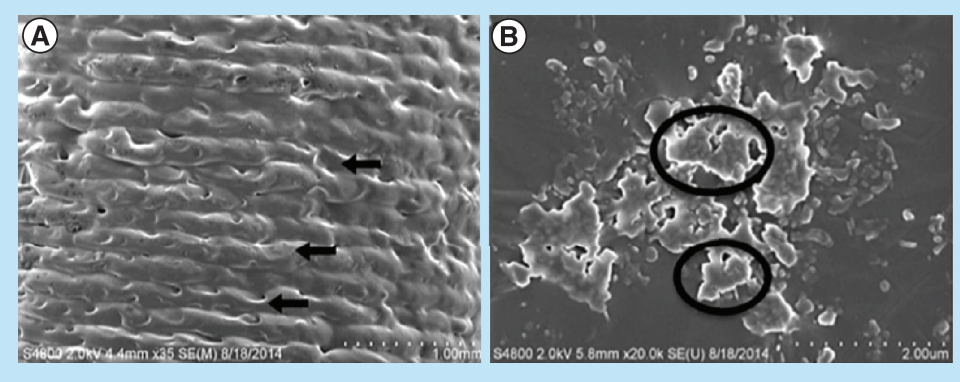

Figure 2. 3D-printed antibiotic containing catheter. Scanning electron microscope images of gentamicin-laden 3D-printed catheters. (A \& B) Multiple amorphous defects seen at $35 \times$ magnification suggest gentamicin incorporation into the catheter structure (by lack of a normal uniform filament structure) (A, arrows). This is confirmed at 20,000 $\times$ magnification, which highlights the amorphous configuration of gentamicin (B, circles). Reproduced with permission from [10] (c) Elsevier (2018).

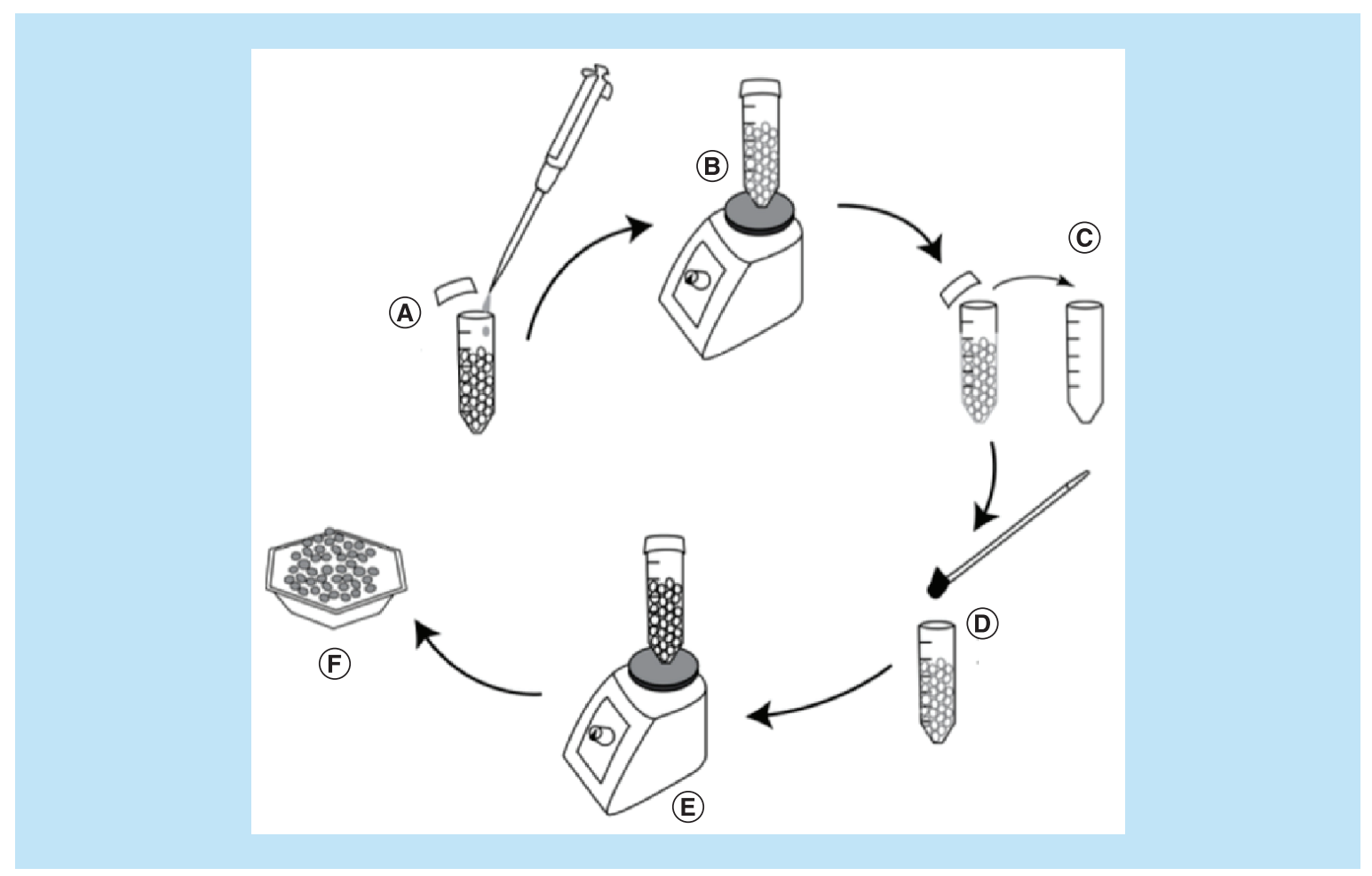

Figure 3. The process of coating polycaprolactone pellets with drugs, including antibiotics. (A) Coating oil is added; (B) tube is vortexed; (C) pellets are transferred to a new tube; (D) drugs are added; (E) tube is vortexed; (F) coated pellets are removed.

Reproduced with permission from [38], (c) PLoS ONE (2017) on an Attribution 4.0 International (CC-BY 4.0) license.

technique. In an early report using FDM to incorporate antibiotics into 3D-printed constructs, an oil coating method was used to surface coat polylactic acid (PLA) polymer pellets with antibiotics, extruded them as filaments of required dimensions and used them to 3D print into constructs (Figure 3) [9].

To avoid heating leading to decreased bioactivity of incorporated antibiotics or antimicrobial agents, investigators have used other techniques such as surface coating or pressure-based extrusion methods. Boyer et al., used singledip coating method to surface coat penicillin onto the surface of 3D-printed nasal stents [13]. Shao et al. [25] used pneumatic extrusion-based bioprinter to load silver phosphate into polycaprolactone polymer along with lidocaine 
to restore irregular bone defect. Visscher et al. [18] used 'solution drop-loading' technique to infuse cefazolin on to the 3D-printed polycaprolactone constructs.

There are, however, limited data regarding the effects of incorporating antibiotics into prints and the subsequent effect on the mechanical stability of the 3D-printed construct. One FDM-based study showed the mechanical properties of 3D-printed constructs with antibiotics were significantly decreased when compared with control constructs [37]. In contrast, a prior study using the inkjet 3D printing technique study demonstrated no change in the mechanical properties of antibiotic-containing constructs compared with those without antibiotics [2]. From this, inkjet printing may be advantageous but is limited by material variety. To our knowledge, there is no data on the mechanical properties of antibiotic-impregnated 3D-printed constructs using stereolithography. In stereolithography-based studies, added nanoparticles did not hinder [25,26] and actually strengthened [25] the mechanical properties of the 3D-printed modified construct; however, antibiotic drugs were not incorporated, but rather nanoparticles with antimicrobial properties.

\section{Summary of current studies: in vitro data}

Table 2 summarizes published antibiotic and antimicrobial-impregnated or containing 3D-printed construct in vitro studies. The use of $3 \mathrm{D}$ printing technology to impregnate drugs into 3D-printed constructs was attempted as early as 1996 by Wu et al. [39]. However, the earliest reported study of impregnating an antibiotic into a 3D-printed implant was reported by Huang et al. in 2007. In that study, they used inkjet 3D printing technology to incorporate levofloxacin in PLA constructs [2]. In a subsequent early report [8], nitrofurantoin was mixed with PLA in order to produce antibiotic-impregnated 3D-printed discs, which showed in vitro inhibition of Staphylococcus aureus biofilm formation. Weisman et al. [9] extruded gentamicin and methotrexate with PLA into antibiotic-impregnated PLA filaments. Using FDM, gentamicin was 3D printed into disc, bead and catheter constructs. The gentamicinladen polylactic acid filaments and discs subsequently showed bacterial inhibition of growth when placed into broth cultures. Similar subsequent experiments reported the same FDM techniques to incorporate gentamicin into antibiotic-impregnated 3D-printed catheters and meshes [10,11]. In a subsequent 3D-printed catheter study, Weisman et al. [10] printed larger catheter constructs impregnated with gentamicin and showed favorable elution profiles over time. Ballard et al. [11] fabricated antibiotic-impregnated surgical meshes and showed their antimicrobial effects in broth culture. Ballard et al. used the same technique to load antibiotics into the polymers and 3D print surgical hernia meshes (Figures 4 \& 5) [11].

Vancomycin, penicillin, levofloxacin and tobramycin have also been incorporated into 3D-printed constructs. Zhou et al. [12] designed a novel composite 3D-printed scaffold impregnated with vancomycin microspheres and demonstrated its drug-eluting capabilities in an in vitro model. In their study, vancomycin microspheres were produced by a non-3D printing technique then placed in solution forming a suspension of the antibiotic microsphere. Subsequently, a polydopamine-coated 3D-printed polylcaprolactone scaffold was placed in the vancomycin microsphere suspension, allowing the microspheres to become impregnated on the polydopamine-polylcaprolactone scaffold. The antibiotic-impregnated construct showed vancomycin release up to the tested duration of 4 weeks in the in vitro model. A nasal support 3D-printed construct study incorporated penicillin into the nasal stent PLA construct and demonstrated inhibition of Escherichia coli growth [13].

$3 \mathrm{D}$ printing antibiotic-impregnated construct studies have also incorporated nondrug chemical elements with antimicrobial properties. Boyer et al. [14] incorporated iodine into 3D-printed constructs, namely model vascular stents. The iodine-impregnated 3D-printed constructs suppressed E. coli and S. aureus growth in human blood agar disk diffusion assays. The incorporation of iodine and other radiopaque compounds into 3D printed constructs has also been the focus of additional 3D-printed implant studies [40,41]. Muwaffak et al. [15] incorporated antimicrobial copper, zinc and silver elements into polylcaprolactone filaments and 3D printed these metal-impregnated filaments as wound dressings. Silver-polylcaprolactone and copper-polylcaprolactone showed more antimicrobial properties against $S$. aureus compared with zinc-impregnated constructs.

\section{Summary of current studies: animal data}

Previously reported antibiotic-impregnated 3D-printed implant studies with animal data [5-7] studied murine [5,7] and rabbit [6] models of osteomyelitis. Inzana et al. [5] used an inkjet 3D printing process to create calcium phosphate scaffolds to create scaffolds impregnated with various configurations of vancomycin and rifampin, and compared the antibacterial effects compared with vancomycin-impregnated bone cement. Rifampin interferes with the formation of bone cement, accounting for the dual versus single antibiotic comparison in that study. The dual 


\begin{tabular}{|c|c|c|c|}
\hline 3DP type & Materials used & Application & Ref. \\
\hline \multirow[t]{4}{*}{ Inkjet } & $\begin{array}{l}\text { Antibiotic or antimicrobial: levofloxacin and rifampicin } \\
\text { Polymer: PLA powder + acetone binder }\end{array}$ & $\begin{array}{l}\text { Implantable devices for prophylaxis and treatment of } \\
\text { bone diseases }\end{array}$ & {$[2,3]$} \\
\hline & $\begin{array}{l}\text { Antibiotic or antimicrobial: kanamycin, tetracycline and } \\
\text { polymyxin B } \\
\text { Polymer: PLA }\end{array}$ & $\begin{array}{l}\text { Portable 3D printer system for diagnosis and treatment } \\
\text { of multidrug-resistant bacteria }\end{array}$ & [4] \\
\hline & $\begin{array}{l}\text { Antibiotic or antimicrobial: vancomycin, rifampin } \\
\text { Ceramic: powder-calcium phosphate. } \\
\text { Binder: phosphoric acid + tween } 80\end{array}$ & $\begin{array}{l}\text { Bioceramics to treat implant-associated bone infection. } \\
\text { Note: included animal data }\end{array}$ & [5] \\
\hline & $\begin{array}{l}\text { Antibiotic or antimicrobial: levofloxacin and tobramycin } \\
\text { Polymer: PLA }\end{array}$ & $\begin{array}{l}\text { Multidrug implant for chronic osteomyelitis. } \\
\text { Note: included animal data }\end{array}$ & [6] \\
\hline \multirow[t]{15}{*}{$\begin{array}{l}\text { Fused deposition } \\
\text { modeling }\end{array}$} & $\begin{array}{l}\text { Antibiotic or antimicrobial: tobramycin } \\
\text { Polymer: PCL/PLGA composite }\end{array}$ & $\begin{array}{l}\text { Scaffolds to treat chronic osteomyelitis. } \\
\text { Note: included animal data }\end{array}$ & [7] \\
\hline & $\begin{array}{l}\text { Antibiotic or antimicrobial: nitrofurantoin } \\
\text { Polymer: PLA }\end{array}$ & $\begin{array}{l}\text { Fabricate antibacterial 3DP material to prevent biofilm } \\
\text { formation on medical devices }\end{array}$ & [8] \\
\hline & $\begin{array}{l}\text { Antibiotic or antimicrobial: gentamicin sulfate } \\
\text { Polymer: PLA }\end{array}$ & $\begin{array}{l}\text { Fabricate 3DP bioactive discs, beads, catheter constructs } \\
\text { and anti-infective surgical meshes }\end{array}$ & [7-11] \\
\hline & $\begin{array}{l}\text { Antibiotic or antimicrobial: vancomycin } \\
\text { Polymer: PCL + polydopamine + PLGA }\end{array}$ & $\begin{array}{l}\text { Novel composite material for the prevention and } \\
\text { treatment of bone infections }\end{array}$ & [12] \\
\hline & $\begin{array}{l}\text { Antibiotic or antimicrobial: penicillin } \\
\text { Polymer: PLA }\end{array}$ & Nasal stents inhibition bacterial growth & [13] \\
\hline & $\begin{array}{l}\text { Antibiotic or antimicrobial: iodine } \\
\text { Polymer: PLA }\end{array}$ & Anti-infective vascular stents & [14] \\
\hline & $\begin{array}{l}\text { Antibiotic or antimicrobial: copper, zinc, silver } \\
\text { Polymer: PCL }\end{array}$ & $\begin{array}{l}\text { Metal-impregnated filaments for anti-infective wound } \\
\text { dressing }\end{array}$ & [15] \\
\hline & $\begin{array}{l}\text { Antibiotic or antimicrobial: tetracycline } \\
\text { Polymer: PLA }\end{array}$ & $\begin{array}{l}\text { Drug-eluting orthopedic liner for periprosthetic joint } \\
\text { infection treatment }\end{array}$ & [16] \\
\hline & $\begin{array}{l}\text { Antibiotic or antimicrobial: tobramycin, nitrofurantoin } \\
\text { Polymers: PLA and PMMA }\end{array}$ & Constructs to treat osteomyelitis & [17] \\
\hline & $\begin{array}{l}\text { Antibiotic or antimicrobial: cefazolin } \\
\text { Polymer: PCL }\end{array}$ & $\begin{array}{l}\text { Cefazolin was coated on the 3DP PCL constructs using } \\
\text { 'solution drop-loading' technique. } \\
\text { 3D-printed scaffolds for local delivery of drugs }\end{array}$ & [18] \\
\hline & $\begin{array}{l}\text { Antibiotic or antimicrobial: quaternary ammonium, } \\
\text { chitosan } \\
\text { Polymer: PLGA }\end{array}$ & Anti-infective osteoconductive composite scaffold & [19] \\
\hline & $\begin{array}{l}\text { Antibiotic or antimicrobial: maleic anhydride-grafted } \\
\text { polylactide, chitosan } \\
\text { Polymer: PLA }\end{array}$ & Antibacterial and cytocompatible material & [20] \\
\hline & $\begin{array}{l}\text { Antibiotic or antimicrobial:silver-graphene oxide } \\
\text { Polymer: } \beta \text {-tricalcium phosphate }\end{array}$ & Antibacterial and osteogenic scaffolds & [21] \\
\hline & $\begin{array}{l}\text { Antibiotic or antimicrobial: copper } \\
\text { Polymer: PLA }\end{array}$ & PLACTIVE ${ }^{T M}$ filament for antibacterial prosthetics & [22] \\
\hline & $\begin{array}{l}\text { Antibiotic or antimicrobial: } \mathrm{Ag}_{3} \mathrm{PO}_{4} \\
\text { Polymer: } \mathrm{PCL}\end{array}$ & $\begin{array}{l}\text { Combinatorial drug scaffolds with antibacterial effects } \\
>6 \text { days }\end{array}$ & [23] \\
\hline \multirow[t]{4}{*}{ Stereolithography } & $\begin{array}{l}\text { Antibiotic or antimicrobial: positively charged } \\
\text { quaternary ammonium compounds } \\
\text { Polymer: diurethanedimethacrylate/glycerol } \\
\text { dimethacrylate }\end{array}$ & $\begin{array}{l}\text { Prevent biomaterial-associated infections for intraoral } \\
\text { appliances and dental restorations }\end{array}$ & [24] \\
\hline & $\begin{array}{l}\text { Antibiotic or antimicrobial: silver-halloysite } \\
\text { Polymer: methacrylate resin }\end{array}$ & $\begin{array}{l}\text { Dental resins with sustained antibacterial effect. } \\
\text { Note: bioprinting technology was used }\end{array}$ & [25] \\
\hline & $\begin{array}{l}\text { Antibiotic or antimicrobial: quaternary ammonium } \\
\text { Polymer: methacrylate resin }\end{array}$ & $\begin{array}{l}\text { Antibacterial surface additive for medical and dental } \\
\text { devices }\end{array}$ & [26] \\
\hline & $\begin{array}{l}\text { Antibiotic or antimicrobial: silver-halloysite } \\
\text { Polymer: methacrylate resin }\end{array}$ & Dental resins with sustained antibacterial effect & [27] \\
\hline
\end{tabular}

drug-impregnated 3D-printed scaffold significantly reduced bacterial burden compared with the single antibiotic bone cement in mice with implant-induced osteomyelitis [5]. Wu et al. [6] used a similar inkjet process to 3D print a multilayered complex cylinder construct impregnated with levofloxacin and tobramycin in a concentric and stacked manner with superficial to deep layers including levofloxacin, tobramycin, levofloxacin and tobramycin, 

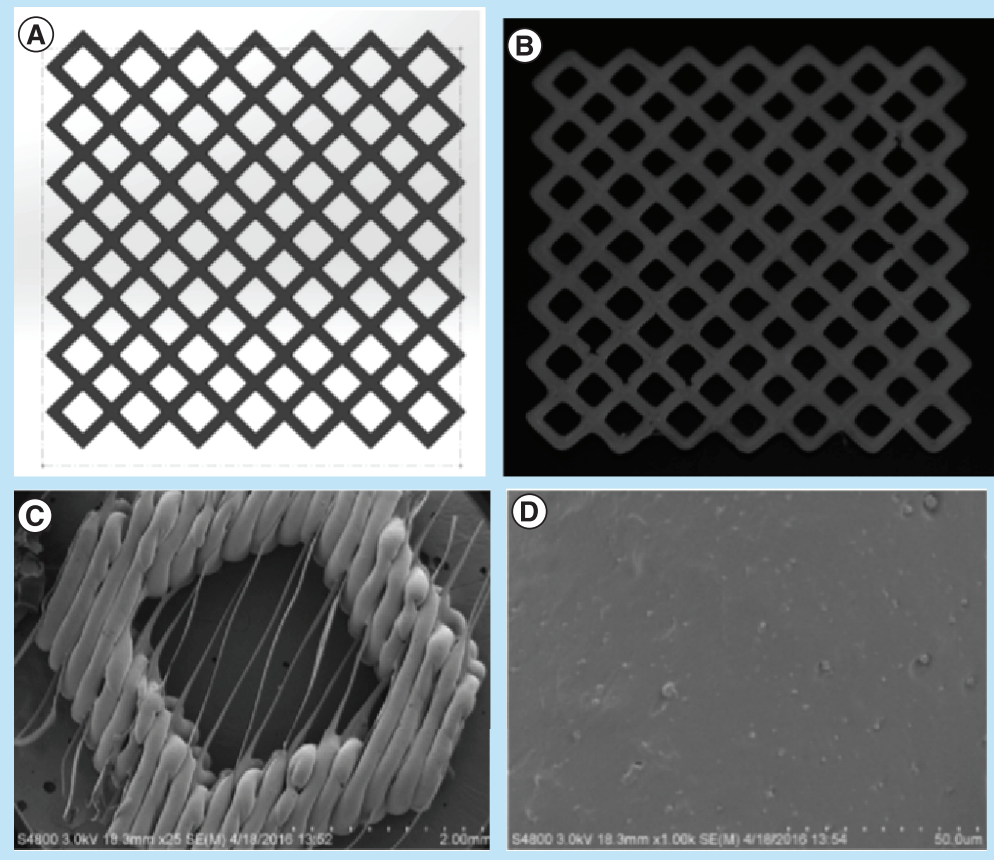

Figure 4. 3D-printed antibiotic containing mesh. (A) Digital, computer-aided design, 3D mesh model. (B) Photograph of 3D-printed mesh construct. (C) Scanning electron microscopy image at 253 magnification showing individual mesh unit/fibers. (D) Scanning electron microscopy at 1000× magnification showing embedded bioactive gentamicin powder.

Reproduced with permission from [11] (c) Elsevier (2017).
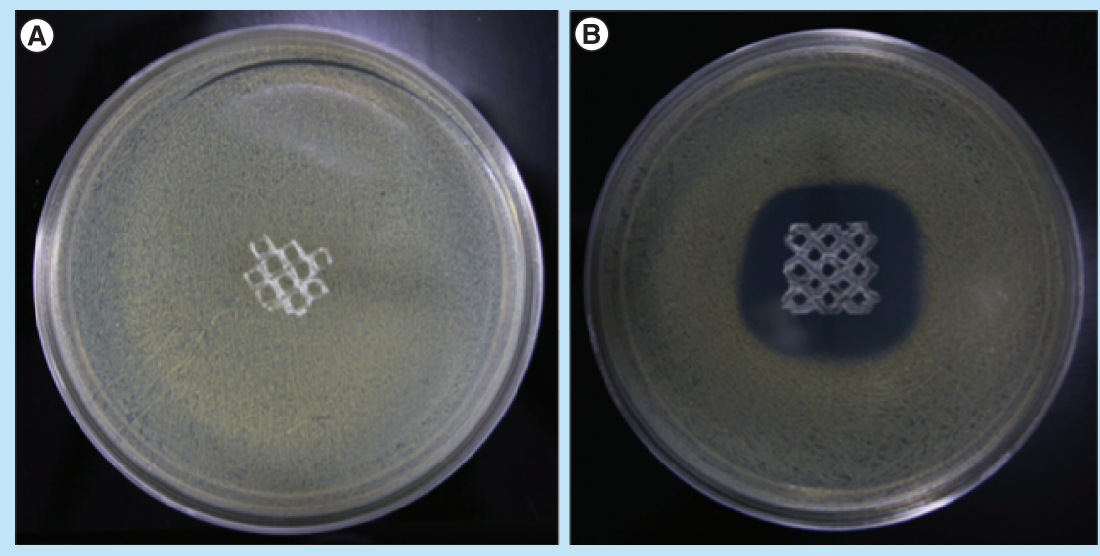

Figure 5. Staphylococcus aureus bacterial culture plates after $24 \mathrm{~h}$ of inoculation. The control mesh (A) has no effect on bacterial growth, while the gentamicin-laden mesh (B) shows a zone of inhibition.

Reproduced with permission from [11] (C) Elsevier (2017).

sequentially. Rabbits received bacterial surgical implantation to induce osteomyelitis. After an induction period and subsequent confirmation of osteomyelitis, the rabbits were debrided and the experimental group had the novel 3D-printed antibiotic implant at the time of debridement. All experimental rabbits failed to develop recurrent osteomyelitis, whereas the rabbits that did not receive the 3D-printed implant did [6]. To our knowledge, only one study has reported a heat-based 3D printing technique in animal data [7]. In that study, FDM 3D printing was used to fabricate a tobramycin-impregnated 3D-printed scaffold, which in in vitro showed drug release up to 51 days. 
In the in vivo testing with rats with induced osteomyelitis, rats that had the antibiotic-impregnated 3D-printed scaffold had improvement in their soft tissue and osseous infection whereas rats with bland scaffolds (no antibiotics) had persistence or worsening infection [7].

\section{Challenges for use in patient care}

Although there are many potential benefits of creating 3D-printed implants and instruments, if this technology is to be beneficial in future animal and human studies, regulatory considerations, drug concentration and required facilities must be overcome prior to their implementation in patient care. The US FDA has released a number of documents and peer-reviewed publications regarding their perspective on regulation of $3 \mathrm{D}$ printing in medicine, including implants $[42,43]$. In brief, 3D-printed implants would qualify as a class III device, which would require an approval process. This may be either through $510(\mathrm{k})$ process where a substantial equivalent exists or a premarket approval pathway. The former approval process typically does not require clinical trials whereas the latter does typically require clinical trials $[1,44,45]$. Detailed summaries of interpreting the FDA's recommendation have been summarized elsewhere $[1,44,45]$. Additional recommendation statements of nonimplantable regulatory considerations of anatomic models and surgical guides have been published as a consensus statement from the Radiological Society of North America 3D Printing Special Interest Group [46].

The potential benefits of producing 3D-printed implants impregnated with antibiotics include customizing implants to patient-specific anatomy from medical imaging and tailoring drug concentrations to patient size and the planned site of implantation [1,11]. However, increasing antibiotic concentration may decrease the mechanical stability of the implant and have a questionable benefit, if any, to the implanted tissue. Studies aiming on varying concentration versus a fixed concentration of drug would be helpful to see if increased drug concentration has benefits to the target tissue. These limitations may obviate the potential benefits of 3D-printed antibioticimpregnated implants.

Establishing or modifying facilities to produce sterile or sterilizable 3D-printed implants impregnated with antibiotics may present logistical challenges. Whereas medical 3D printing labs that produce anatomic models may not need special ventilation accommodations such as fume hoods, but this is or an equivalent is needed when working with heating 3D-printed base materials coated or mixed with drug. Sterilizing the implant can be accomplished by establishing a sterile or substerile 3D printer and 3D printing space where it is printed sterile, heating by autoclave after printing, or by chemical sterilization (e.g., glutaraldehyde) [1]. Allocating a space for sterile printing is logistically difficult and the latter two techniques may decrease the desired effect or concentration of the impregnated antibiotic.

\section{Conclusion}

There are a number of potential advantages of using 3D-printed implants impregnated with antibiotics including biodegradable materials that expose new layers of drug to the environment over time, drug concentration and distribution customization, and patient customization based on medical imaging. There have been a number of promising in vitro investigations [2-4,8-18] that require further substantial in vivo animal and human studies. 3Dprinted drug-impregnated implants are in early development and future studies will determine if they will be useful in delivering patient-specific medicine.

\section{Future perspective}

Animal and human investigations aimed at demonstrating efficacy of 3D-printed antibiotic-impregnated implants are needed to supplement the few pre-existing animal studies [5-7] and demonstrate the clinical feasibility of using these constructs. Moreover, the previously published animal studies all focused on osteomyelitis; other disease processes should be investigated. The most pertinent topic that needs to be addressed in these studies is assessing whether or not the 3D-printed implants impregnated with drugs have mechanical stability equivocal to commercially available implants. In vitro data would suggest that localized drug delivery should be feasible [2-4,8-19], but data comparing 3D-printed drug-impregnated implant site infection with 3D-printed implants without drugs or commercial products with and without systemic antibiotics would be an ideal study design. As summarized in Table 2, three different 3D printing techniques have been described. Head-to-head comparison of the three different techniques with focus on their drug release profile and mechanical properties would be beneficial for future studies to determine the optimal 3D printing technique and delivery mechanism. Integrating drugs into bioprinted constructs or hybrid bioactive printing with cellular coatings may also offer advantages to traditional 
implants [27]. Newer techniques, such as 3D continuous liquid interface production may also be of interest, as the method allows for faster production rates and has shown controllable drug-release profiles from medical devices [47]. Overall, all of the summarized 3D printing methods hold tremendous potential in medicine; however, are still in their infancy in terms of clinical efficacy.

\section{Executive summary}

\section{Background}

- Bioactive printing and bioactive 3D printing are terms sometimes used to describe the process of implanted drugs, including antibiotics, into 3D-printed implants and constructs.

- At writing, there are no antibiotic-impregnated 3D-printed implant studies in humans.

Rationale of incorporating antibiotics into 3D-printed constructs

- Antibiotic-impregnated bone cements are used clinically in patient care and serve as an example why pursuing custom 3D-printed antibiotic implants may be of therapeutic and clinical interest.

- One potential advantage of 3D-printed construct impregnated with antibiotics is that the drug is present at deep layers that are not initially exposed to the tissue upon initial implant, are gradually exposed as superficial layers dissolve.

- Other advantages of 3D printing include the layer-by-layer fabrication leads to increased surface area for drug distribution and the ability to rapidly fabricate constructs based on patient-specific anatomy derived from medical imaging.

Technical considerations

- The earliest reports used inkjet printing, whereas the majority of subsequent studies have used fused deposition modeling (FDM), and a few studies have used stereolithography.

- Inkjet and stereolithography printing as methods to incorporate antibiotics into 3D-printed constructs are limited by choice of materials.

- FDM can use a wide array of materials to incorporate antibiotics into 3D-printed constructs.

- FDM may have detrimental effects on thermolabile drugs, and stereolithography may have detrimental effects on ultraviolet sensitive drugs.

- We illustrate the preferred method of incorporating antibiotics into 3D-printed constructs using FDM.

Summary of current studies: in vitro data \& animal data

- Majority of antibiotic-impregnated 3D-printed implant studies are in vitro.

- The few animal studies focus on osteomyelitis models.

- Prior studies using 3D printing to incorporate antibiotics or antimicrobials into constructs and implants are summarized in narrative and tabular form (Table 2).

Challenges for use in patient care

- The US FDA's and societal opinion on 3D-printed constructs in medicine as medical devices and their potential regulations are briefly presented.

- 3D-printed antibiotic-containing implants have not been tested in humans. Their potential future clinical use will depend on future studies demonstrating a measurable benefit.

- Challenges associated with sterilizing antibiotic containing 3D-printed implants are summarized.

Future perspective

- Animal studies focusing on diseases different than osteomyelitis, human studies and studies using different 3D printing techniques are all warranted.

Financial \& competing interests disclosure

DH Ballard receives salary support from NIH TOP-TIER grant T32-EB021955. JA Weisman and DK Mills are co-inventors on a patent application that related to methods described in this paper: 'Methods and Devices for Three-Dimensional Printing or Additive Manufacturing of Bioactive Medical Devices', application number US14822275. The authors have no other relevant affiliations or financial involvement with any organization or entity with a financial interest in or financial conflict with the subject matter or materials discussed in the manuscript apart from those disclosed.

No writing assistance was utilized in the production of this manuscript. 


\section{References}

Papers of special note have been highlighted as: $\bullet$ of interest

1. Ballard DH, Trace AP, Ali S et al. Clinical applications of 3D printing: primer for radiologists. Acad. Radiol. 25(1), 52-65 (2008).

- Provides an overview of clinical uses of 3D-printed constructs in patient care along with uses of implants in patient care and translational studies. Prior publication of one of the authors of the present work.

2. Huang W, Zheng Q, Sun W, Xu H, Yang X. Levofloxacin implants with predefined microstructure fabricated by three-dimensional printing technique. Int. J. Pharm. 339(1-2), 33-38 (2007).

- To our knowledge, first publication detailing a 3D-printed antibiotic-impregnated implant construct. The authors use inkjet printing. Well-designed study with relevant discussion and context.

3. Wu W, Zheng Q, Guo X, Sun J, Liu Y. A programmed release multi-drug implant fabricated by three-dimensional printing technology for bone tuberculosis therapy. Biomed. Mater. 4(6), 065005 (2009).

4. Glatzel S, Hezwani M, Kitson Philip J, Gromski Piotr S, Schürer S, Cronin L. A portable 3D printer system for the diagnosis and treatment of multidrug-resistant bacteria. Chem 1(3), 494-504 (2016).

5. Inzana JA, Trombetta RP, Schwarz EM, Kates SL, Awad HA. 3D printed bioceramics for dual antibiotic delivery to treat implant-associated bone infection. Eur. Cell Mater. 30, 232-247 (2015).

6. Wu W, Ye C, Zheng Q, Wu G, Cheng Z. A therapeutic delivery system for chronic osteomyelitis via a multi-drug implant based on three-dimensional printing technology. J. Biomater. Appl. 31(2), 250-260 (2016).

7. Shim J-H, Kim M-J, Park JY et al. Three-dimensional printing of antibiotics-loaded poly-e-caprolactone/poly(lactic-co-glycolic acid) scaffolds for treatment of chronic osteomyelitis. Tissue Eng. Regenerative Med. 12(5), 283-293 (2015).

8. Sandler N, Salmela I, Fallarero A et al. Towards fabrication of 3D printed medical devices to prevent biofilm formation. Int. J. Pharm. 459(1-2), 62-64 (2014).

9. Weisman JA, Nicholson JC, Tappa K, Jammalamadaka U, Wilson CG, Mills DK. Antibiotic and chemotherapeutic enhanced three-dimensional printer filaments and constructs for biomedical applications. Int. J. Nanomedicine 10, 357-370 (2015).

- First report of the author's preferred technique of incorporating antibiotics into 3D-printed constructs with fused deposition modeling. Prior publication of several authors of the present work.

10. Weisman JA, Ballard DH, Jammalamadaka U et al. 3D printed antibiotic and chemotherapeutic eluting catheters for potential use in interventional radiology. Acad. Radiol. 26(2), 270-274 (2019).

11. Ballard DH, Weisman JA, Jammalamadaka U, Tappa K, Alexander JS, Griffen FD. Three-dimensional printing of bioactive hernia meshes: in vitro proof of principle. Surgery 161(6), 1479-1481 (2017).

12. Zhou Z, Yao Q, Li L et al. Antimicrobial activity of 3D-printed poly( $\varepsilon$-caprolactone) (PCL) composite scaffolds presenting vancomycin-loaded polylactic acid-glycolic acid (PLGA) microspheres. Med. Sci. Monit. 24, 6934-6945 (2018).

13. Boyer CJ, Woerner JE, Galea C et al. Personalized bioactive nasal supports for postoperative cleft rhinoplasty. J. Oral Maxillofac. Surg. 76(7), 1562.e1-1562.e5 (2018).

14. Boyer CJ, Ballard DH, Weisman JA et al. Three-dimensional printing antimicrobial and radiopaque constructs. 3D Printing Additive Manufacturing 5, 29-35 (2018).

15. Muwaffak Z, Goyanes A, Clark V, Basit AW, Hilton ST, Gaisford S. Patient-specific 3D scanned and 3D printed antimicrobial polycaprolactone wound dressings. Int. J. Pharm. 527, 161-170 (2017).

16. Kim TWB, Lopez OJ, Sharkey JP, Marden KR, Murshed MR, Ranganathan SI. 3D printed liner for treatment of periprosthetic joint infections. Med. Hypotheses 102, 65-68 (2017).

17. Mills DK, Jammalamadaka U, Tappa K, Weisman J. Studies on the cytocompatibility, mechanical and antimicrobial properties of 3D printed poly(methyl methacrylate) beads. Bioact. Mater. 3, 157-166 (2018).

18. Visscher LE, Dang HP, Knackstedt MA, Hutmacher DW, Tran PA. 3D printed polycaprolactone scaffolds with dual macro-microporosity for applications in local delivery of antibiotics. Mater. Sc. Eng. C Mater. Biol. Appl. 87, 78-89 (2018).

19. Yang Y, Yang S, Wang Y et al. Anti-infective efficacy, cytocompatibility and biocompatibility of a 3D-printed osteoconductive composite scaffold functionalized with quaternized chitosan. Acta Biomater. 46, 112-128 (2016).

20. Wu CS. Modulation, functionality, and cytocompatibility of three-dimensional printing materials made from chitosan-based polysaccharide composites. Mater. Sci. Eng. C Mater Biol Appl. 69, 27-36 (2016).

21. Zhang Y, Zhai D, Xu M et al. 3D-printed bioceramic scaffolds with antibacterial and osteogenic activity. Biofabrication 9(2), 025037 (2017).

22. Zuniga MJ. 3D printed antibacterial prostheses. Appl. Sci. 8(9), 1651 (2018).

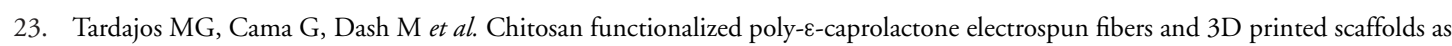
antibacterial materials for tissue engineering applications. Carbohydr. Polymers 191, 127-135 (2018).

24. Chen S, Yang J, Jia Y-G, Lu B, Ren L. A study of 3D-printable reinforced composite resin: PMMA modified with silver nanoparticles loaded cellulose nanocrystal. Materials 11(12), E2444 (2018). 
25. Shao J, Ma J, Lin L et al. Three-dimensional printing of drug-loaded scaffolds for antibacterial and analgesic applications. Tissue Eng. Part C Methods 25(4) 222-231 (2019).

26. Yue J, Zhao P, Gerasimov JY et al. 3D-printable antimicrobial composite resins. Adv. Funct. Mater. 25, 6756-6767 (2015).

27. Sa L, Kaiwu L, Shenggui $\mathrm{C}$ et al. 3D printing dental composite resins with sustaining antibacterial ability. J. Mater. Sci. 54, 3309-3318 (2019).

28. Vanepps JS, Younger JG. Implantable device-related infection. Shock 46(6), 597-608 (2016).

29. Schierholz JM, Beuth J. Implant infections: a haven for opportunistic bacteria. J. Hosp. Infect. 49(2), 87-93 (2001).

30. Calhoun JH, Mader JT. Antibiotic beads in the management of surgical infections. Am. J. Surg. 157, 443-449 (1989).

31. Lee DK. Drug-eluting stent in malignant biliary obstruction. J. Hepatobiliary Pancreat. Surg. 16(5), 628-632 (2009).

32. Buchholz HW, Engelbrecht H. [Depot effects of various antibiotics mixed with Palacos resins]. Chirurg 41, 511-515 (1970).

33. Tappa K, Jammalamadaka U. Novel biomaterials used in medical 3D printing techniques. J. Funct. Biomater. 9, 17 (2018).

34. Jammalamadaka U, Tappa K. Recent advances in biomaterials for 3D printing and tissue engineering. J. Funct. Biomater. 9, 22 (2018).

35. Hodgdon T, Danrad R, Patel MJ et al. Logistics of three-dimensional printing: primer for radiologists. Acad. Radiol. 25(1), 40-51 (2018).

36. Maleksaeedi S, Wang JK, El-Haije A et al. Toward 3D printed bioactive titanium scaffolds with bimodal pore size distribution for bone ingrowth. Procedia CIRP 5, 158-163 (2013).

37. Tappa K, Jammalamadaka U, Weisman AJ et al. 3D printing custom bioactive and absorbable surgical screws, pins, and bone plates for localized drug delivery. J. Funct. Biomater. 10(2), E17 (2019).

- Shows that in fused deposition modeling 3D printing, the addition of antibiotics (and chemotherapeutics) significantly weakened tested mechanical proprerties of the 3D-printed constructs. Prior publication of several authors of the present work.

38. Tappa K, Jammalamadaka U, Ballard DH et al. Medication eluting devices for the field of OBGYN (MEDOBGYN): 3D printed biodegradable hormone eluting constructs, a proof of concept study. PLoS ONE 12(8), e0182929 (2017).

39. Wu BM, Borland SW, Giordano RA, Cima LG, Sachs EM, Cima MJ. Solid free-form fabrication of drug delivery devices. J. Control. Rel. 40, 77-87 (1996).

40. Boyer CJ, Boktor M, Samant H et al. 3D printing for bio-synthetic biliary stents. Bioengineering 6(16), E16 (2019).

41. Ballard DH, Jammalamadaka U, Tappa $\mathrm{K}$ et al. 3D printing of surgical hernia meshes impregnated with contrast agents: in vitro proof of concept with imaging characteristics on computed tomography. 3D Print. Med. 4(1), 13 (2018).

42. Di Prima M, Coburn J, Hwang D, Kelly J, Khairuzzaman A, Ricles L. Additively manufactured medical products - the FDA perspective. 3D Print. Med. 2(1) doi:10.1186/s41205-016-0005-9 (2016).

43. Ricles LM, Coburn JC, Di Prima M, Oh SS. Regulating 3D-printed medical products. Sci. Transl. Med. 10 (461) eaan6521 (2018).

- The authors are US FDA's employees and provide an overview of the FDA's perspective of regulating 3D-printed medical products.

44. Christensen A, Rybicki FJ. Maintaining safety and efficacy for 3D printing in medicine. 3D Print. Med. 3(1), 1 (2017).

- Provides a robust framework of how 3D-printed constructs including implants may be regarded as implants according to the FDA, and suggests classifications of different tiers of 3D-printed constructs as devices. In contradiction to the previous publication, the authors are not FDA employees. However, they are considerable 3D printing experts and the authors of the present work would strongly recommend anyone intending to use 3D-printed constructs in patient care to read this article.

45. Morrison RJ, Hollister SJ, Niedner MF et al. Mitigation of tracheobronchomalacia with 3D-printed personalized medical devices in pediatric patients. Sci. Transl. Med. 7(285), 285 ra64 (2015).

46. Chepelev L, Wake N, Ryan J et al. Radiological Society of North America (RSNA) 3D printing Special Interest Group (SIG): guidelines for medical 3D printing and appropriateness for clinical scenarios. 3D Print. Med. 4(1), 11 (2018).

- Provides a medical 3D printing society's expert reccomendations on guidelines for medical 3D printing. One of the authors of the present work is a coauthor of this reference.

47. Bloomquist CJ, Mecham MB, Paradzinsky MD et al. Controlling release from 3D printed medical devices using CLIP and drug-loaded liquid resins. J. Control. Rel. 278, 9-23 (2018). 
(

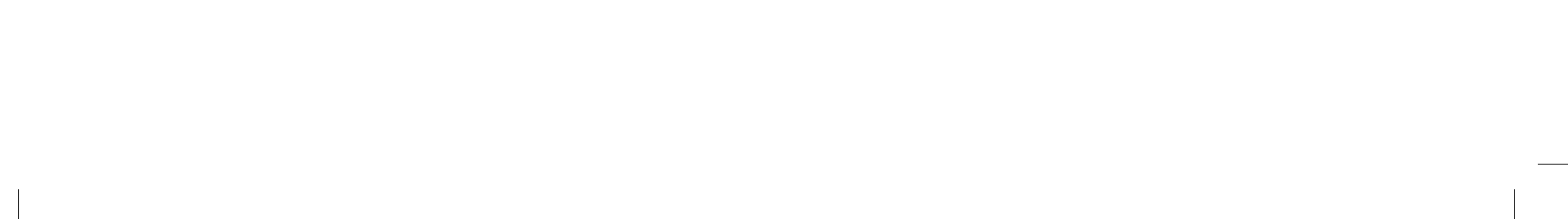

